\title{
NOUVELLE
}

\section{ATP1A2 : \\ un facteur essentiel \\ dans la migraine hémiplégique familiale}

Rhoda Blostein, Laura Segall, J. Jay Gargus
> Il est communément admis que la migraine présente une forte composante génétique. À cet égard, des travaux récents qui ont établi que des for-



Figure 1. Le cycle de réaction de la Na,K-ATPase. Selon le modèle «Albers-Post» abrégé, l'enzyme en $\varepsilon_{1}$ lie le $\mathrm{Na}^{+}$cyT et I'ATP avec haute affinité. L'enzyme est alors phosphorylé sur un résidu aspartate dans la boucle cytoplasmique TM4TM5 (voir Figure 2) menant à l'occlusion des ions de $\mathrm{Na}^{+}$ $\left(\varepsilon_{1} P\right.$. [3Na]). Par suite d'un changement de conformation en $\varepsilon_{2} P$, l'affinité apparente pour le $\mathrm{K}^{+}{ }_{\text {EXT }}$ est nettement augmentée et diminuée pour le $\mathrm{Na}^{+}$tel que ces derniers sont relâchés en faveur de deux $K^{+}{ }_{\varepsilon X T}\left(\varepsilon_{2} P .2 K\right)$. Les ions de $K^{+}$sont alors occlus en $\varepsilon_{2} P$. [2K] qui est rapidement déphosphorylée pour donner $\varepsilon_{2 .}[2 K]$. Les ions de $K^{+}$sont à leur tour relâchés, l'enzyme lie l'ATP (liaison à basse affinité) pour former $\varepsilon_{2}$.ATP qui change rapidement de conformation de sorte que l'enzyme est prêt pour un autre cycle. Le cycle catalytique de cette pompe ionique de type $\mathrm{P}$ implique une translocation de $\mathrm{Na}^{+}$et $\mathrm{K}^{+}$couplée à une hydrolyse ATP impliquant une phosphorylation et une déphosphorylation de la sous-unité catalytique $\alpha$ (résidu aspartyl sur l'enzyme, $\varepsilon$ ) et des transitions conformationnelles de la phospho- et déphosphoenzyme $\left(\varepsilon_{1} P \rightarrow \varepsilon_{2} P\right.$ et $\left.\varepsilon_{1} \rightarrow \varepsilon_{2}\right)$. Ext : extracellulaire; Cyt : cytoplasmique.
R. Blostein, L. Segall :

Départements de biochimie et médecine, Université McGill, Montréal, Québec, Canada et Institut de recherche de l'Hôpital Général de Montréal, 1650, avenue Cedar, Montréal, Québec, H3G 1A4 Canada. J. Jay Gargus : Départements de physiologie, biophysique et pédiatrie, section de Génétique humaine, Université de Californie, Irvine, Californie, États-Unis. rhoda.blostein@mcgill.ca mes autosomiques rares de migraine avec aura, migraine hémiplégique (FHM), sont associées à des mutations faux-sens dans au moins deux gènes, ont ouvert de nouvelles perspectives sur la physiologie de la migraine. Ces deux gènes, CACNAIA qui code la sous-unité $\alpha$ l du canal neuronal de $\mathrm{Ca}^{2+}$ sensible au voltage $\left(\mathrm{Ca}_{\mathrm{V}} 2.1\right)$ et ATPIA2 qui code l'isoforme $\alpha 2$ de la $\mathrm{Na}, \mathrm{K}$-ATPase sont impliqués dans la FHMI et la FHM2 respectivement. À eux deux, ils répondent à la plupart des cas de FHM [1]. Récemment néanmoins, une mutation fauxsens (Gl489K) dans le gène SCNIA du canal ionique du $\mathrm{Na}^{+}$dépendant du voltage neuronal a également été signalée [2]. Il est probable que, considérées conjointement, la FHM et peut-être d'autres formes de migraine résultent d'une déficience du transport normal de cations. Cet article discute la nature des altérations fonctionnelles susceptibles de justifier le phénotype de la FHM2.

\section{La structure de la Na,K-ATPase, diversité de fonctions et d'isoformes}

La Na,K-ATPase catalyse l'échange électrogène, réglé par I'ATP, de trois ions $\mathrm{Na}^{+}$CyT contre deux ions $\mathrm{K}^{+}$EXT à travers la membrane plasmique de presque toutes les cellules animales; elle est essentielle à l'entretien des gradients électrochimiques de cations alcalins qui sont abolis par les canaux ioniques dans la propagation des potentiels d'action [3, 4]. Cette pompe comprend une grande $(\approx 110 \mathrm{kDa})$ sous-unité catalytique $\alpha$ qui possède 10 segments transmembranaires et une sous-unité $\beta$ plus petite, hautement glycosylée, qui assure le repliement et l'ancrage appropriés de la sous-unité $\alpha$ dans la membrane. Quatre isoformes de $\alpha$ et trois de $\beta$ ont été décrites jusqu'ici ; elles sont distribuées de manière tissuet développement-dépendante. Chez les mammifères adultes, $\alpha 2$ se trouve principalement dans le muscle squelettique et le cerveau, en particulier dans les cellules gliales, et dans une moindre mesure dans le cœur, les adipocytes et I'œil. La Figure 1 illustre un cycle de réaction abrégé de la $\mathrm{Na}, \mathrm{K}$-ATPase qui, comme toutes les pompes de type $P$, implique des transitions de conformation des formes déphospho- et phosphorylées de l'enzyme. 


\section{FHM2 associée à des mutations faux-sens dans l'isoforme a2 de la Na,K-ATPase}

Jusqu'ici, plus de 20 allèles du gène $\alpha 2$ ont été relevés chez les membres de familles où sévit la FHM2 (Figure 2). La plupart des allèles sont des mutations faux-sens et, parmi eux, au moins neuf sont bien identifiés; les autres allèles candidats attendent validation. L'analyse fonctionnelle des deux premières mutations rapportées, L764P et W887R [5], suggérait que la maladie résulte d'une haplo-insuffisance puisque ces mutants sont inactifs comme en témoigne leur incapacité à soutenir la croissance de cellules mammifères en culture. Depuis, nous avons clairement montré qu'au moins trois autres mutants, T345A, R689P et M731T, sont fonctionnels et soutiennent la croissance cellulaire, mais affichent une cinétique altérée $[6,7]$.

Ainsi, I'ADNc des formes résistantes à l'ouabaïne des mutants ${ }^{1}$ a été introduit dans des cellules HeLa cultivées en présence d'ouabaïne à $l \mu M$ afin d'inhiber l'activité de la pompe endogène humaine. De même que la forme de type sauvage, celles des mutants soutiennent la croissance des cellules HeLa suggérant que le phénotype de croissance, en présence de ouabaïne [5], n'est pas suffisant pour distinguer les allèles pathogéniques. Nous avons alors procédé à une analyse cinétique détaillée.

Nos résultats montrent que le mutant FHM2 T345A possède un taux de croissance et un renouvellement catalytique similaire à ceux de $\alpha 2$ de type sauvage. L'analyse d'autres paramètres cinétiques [6] suggère que la baisse d'affinité pour le $K^{+}$(doublement de $\left.K_{0.5(K)}\right)$ reflète une augmentation du délestage de $K^{+}$via les réac-

${ }^{1}$ L'introduction des mutations Q116R et N127D à la bordure de la première boucle extracellulaire rend l'isoforme $\alpha 2$ et ses mutants relativement insensibles à l'ouabaïne afin de pouvoir distinguer leur cinétiques de ceux de l'enzyme endogène HeLa [10]. tions $\varepsilon_{2} .2[\mathrm{~K}] \rightarrow \varepsilon_{1} .3 \mathrm{Na}$ (voir Figure 1 ). La modélisation par homologie [7] suggère que $\mathrm{T} 345 \rightarrow \mathrm{A}$ affecte la coordination $\mathrm{du} \mathrm{K}^{+}$en interférant avec le déplacement de TM4 lors des transitions de conformation contribuant à la formation de la poche de liaison des cations.

Inversement, R6890 et M731T entraînent plusieurs changements indicatifs d'un déplacement de l'équilibre $\varepsilon_{1} \leftrightarrow \varepsilon_{2}$ vers $\varepsilon_{1}$ [7] y compris une augmentation de l'affinité pour $\mathrm{K}_{\varepsilon \times \mathrm{T}}^{+}$, une baisse du renouvellement catalytique et une inhibition par l'orthovanadate inorganique, un analogue du phosphate inorganique se liant à $\varepsilon_{2}$. La modélisation structurale [7] prédit que $\mathrm{R} 689 \rightarrow 0$ interfère avec les interactions normales des domaines cytoplasmiques, tandis que $M 73 \mathrm{l} \rightarrow \mathrm{T}$ diminue l'affinité pour le $\mathrm{Mg}^{2+}$ dans la réaction de transfert qui dépend du groupement $\mathrm{Mg}^{2+}$ - phosphoryl.

Ensemble, ces résultats suggèrent que le phénotype FHM est dû à une diminu-

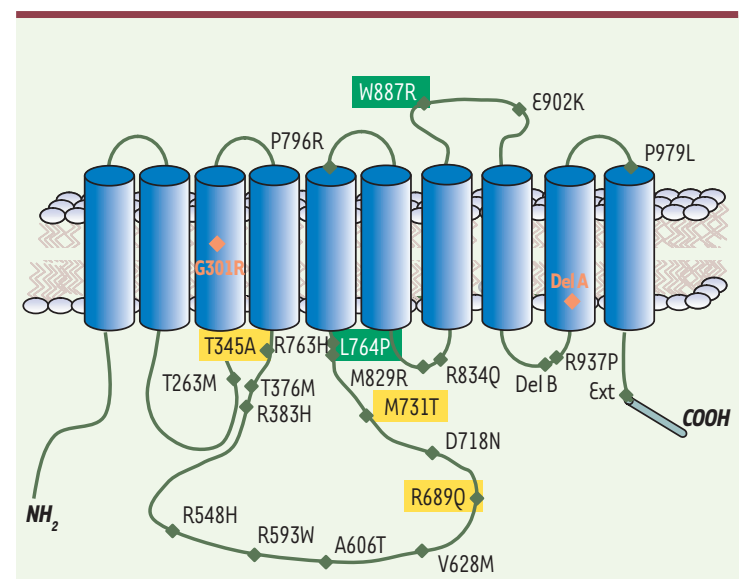

Figure 2. Structure $2 D$ schématique de la sous-unité catalytique Na,K-ATPase à 10 transmembranes montrant des mutations associées à la migraine hémiplégique [9]. Les mutations faux-sens fonctionnellement caractérisées sont surlignées (vert [5], jaune [6,7]). Les mutations de délétion sont Del A (nt2897 et 2898 conduisant à une mutation de changement de phase et l'introduction d'un codon non-sens) et Del B (K935-S940 et insertion de I) et une mutation menant à une extension carboxy-terminale (Ext ; mutation codon non-sens $X 1021 \rightarrow R$ conduisant à l'ajout de 27 résidus à la partie carboxy-terminale). tion de l'activité de la pompe $\alpha 2$ pouvant survenir soit par des mutations causant l'inactivation de la pompe (haplo-insuffisance [5]), une affinité apparente diminuée pour le $\mathrm{K}^{+}$ (T345A), soit par suite d'une baisse du renouvellement catalytique ( $R 6890$ et M731T). La diminution de l'activité entraînerait un délai dans la clairance de $K_{E X T}^{+}$et/ou un traitement/signalisation localisé altéré de $\mathrm{Ca}^{2+}$ secondaire à une activité réduite de l'échangeur $\mathrm{Na}^{+} / \mathrm{Ca}^{2+}$ colocalisé comme on le discute ci-dessous.

Puisque l'isoforme $\alpha 2$ de la $\mathrm{Na}, \mathrm{K}$-ATPase constitue un composant relativement mineur de l'activité totale de Na,K-ATPase dans le cerveau, l'explication la plus probable du phénotype de la maladie est que ces pompes sont confinées à des microdomaines de la membrane plasmique. Par conséquent, cette localisation conduirait à des changements dans les concentrations de cations dans des régions localisées à l'entrée des canaux ioniques et des échangeurs. Des études avec des souris déficientes en $\alpha 2$, montrant un accroissement local de la concentration d'ions $\mathrm{Na}^{+}$et, via un échange de $\mathrm{Na} / \mathrm{Ca}$, une augmentation de la concentration d'ions $\mathrm{Ca}^{2+}$ près du réticulum endoplasmique [8], viennent étayer ce concept. La colocalisation de la sous-unité $\alpha 2$ de la pompe $\mathrm{Na}$,K-ATPase et de l'échangeur $\mathrm{Na} / \mathrm{Ca}$ de la membrane plasmique offre une base séduisante pour proposer que la FHM pourrait être causée par des mutations altérant le $\mathrm{Ca}^{2+}$ intracellulaire dans un microdomaine critique pour la signalisation de $\mathrm{Ca}^{2+}$ et ce, quel que soit le gène impliqué. $\diamond$

ATPIA2:

a key player in familial hemiplegic migraine 


\section{RÉFÉRENCES}

1. Wessman M, Kaunisto M, Kallela M, Palotie A. The molecular genetics of migraine. Ann Med 2004 ; $36: 462-73$.

2. Dichgans M, Freilinger T, Eckstein G. et al. Mutation in the neuronal voltage-gated sodium channel SCN1A in familial hemiplegic migraine. Lancet 2005 ; $366: 371-7$.

3. Jorgensen PL, Kansson KOH, Karlish SJD. Structure and mechanism of $\mathrm{Na}$, K-ATPase : functional sites and their interactions. Ann Rev Physiol 2003 ; 65 : 817-49.
4. Kaplan JH. Biochemistry of $\mathrm{Na}, \mathrm{K}$-ATPase. Ann Rev Biochem 2002; 71: 511-35.

5. De Fusco M, Marconi R, Silvestri L, et al. Haploinsufficiency of ATP1A2 encoding the $\mathrm{Na}^{+} / \mathrm{K}^{+}$ pump alpha 2 subunit associated with familia hemiplegic migraine type 2. Nat Genet 2003; $33: 192-6$.

6. Segall L, Scanzano R, Kaunisto MA, et al. Kinetic alterations due to a missense mutation in the $\mathrm{Na}$, K-ATPase alpha2 subunit cause familial hemiplegic migraine type 2. J Biol Chem 2004 ; 279 : 43692-6.

7. Segall L, Mezzetti A, Scanzano R, et al. Alterations in the alpha2 isoform of $\mathrm{Na}$, K-ATPase associated with

\section{NOUVELle}

\section{LMP-420, \\ un nouvelle approche thérapeutique pour le paludisme cérébral ?}

Samuel C. Wassmer, George J. Cianciolo, Valéry Combes, Georges $\varepsilon$. Grau

> Le paludisme représente la première endémie parasitaire au monde. Elle cause près de 2,5 millions de décès par an, dont une grande majorité d'enfants en bas âge et de femmes enceintes. Le paludisme cérébral (PC) (ou neuropaludisme) est une forme grave de l'infection à Plasmodium falciparum, caractérisé par une accumulation plus importante d'hématies parasitées par P. falciparum, de leucocytes mononucléés et de plaquettes au niveau des microvaisseaux cérébraux, ainsi que par une activation endothéliale systémique et un nombre accru de microparticules (MP) endothéliales circulantes (pour revue, voir [1]).

De nombreuses études ont démontré le rôle clé de certaines cytokines proinflammatoires dans le développement du PC, comme le tumor necrosis factor (TNF) et plus récemment la lymphotoxine $\alpha$ (LT). Ces deux cytokines appartiennent à la même famille, et leur interaction avec le même récepteur, le TNFR2, conduit à l'activation de l'endothélium [2], caractérisée par une augmentation d'expression de molécules d'adhérence, mais aussi une libération de quantités accrues de MP.

Nous avons testé les propriétés antiinflammatoires d'une nouvelle molécule nommée LMP-420 dans un modèle in vitro de lésion cérébrale développé dans notre laboratoire. La stimulation de cellules endothéliales cérébrales humaines par TNF ou LT conduit à une augmentation de l'expression des molécules ICAM-1 (intercellular adhesion molecule 1) et VCAM-1 (vascular cell adhesion molecule 1), deux molécules dont l'expression est plus élevée à la surface de l'endothélium cérébral chez les patients décédés de $P C$ comparativement aux témoins $[3,4]$. Cependant, lorsque les cellules endothéliales sont stimulées en présence de LMP-420, une inhibition significative de cette augmentation d'expression est observée.

ICAM-1 et VCAM-1 étant des récepteurs utilisés par les hématies parasitées par $P$. falciparum lors de la cytoadhérence, nous avons étudié l'impact de cette inhibition sur l'adhérence des hématies familial hemiplegic migraine type 2. Proc Natl Acad Sci USA 2005; 102 : 11106-11.

8. Golovina VA, Song $\mathrm{H}$, James $\mathrm{PF}$, et al. $\mathrm{Na}^{+}$pump alpha 2-subunit expression modulates $\mathrm{Ca}^{2+}$ signaling. Am J Physiol 2003; 284 : C475-86.

9. Riant F, De Fusco M, Aridon P, et al. ATP1A2 mutations in 11 families with familial hemiplegic migraine. Hum Mutat $2005 ; 26: 281$.

10. Jewell $\varepsilon A$, Lingrel JB. Comparison of the substrate dependence properties of the rat $\mathrm{Na}, \mathrm{K}$-ATPase alpha 1, alpha 2, and alpha 3 isoforms expressed in HeLa cells. J Biol Chem 1991 ; 266 : 16925-30.
S.C. Wassmer : Malawi-Liverpool Wellcome Trust Clinical Research Programme, PO Box 30096, Chichiri, Bantyre 3, Malawi. G.J. Cianciolo: Département de Pathologie, Duke University Medical Center, P0 Box 3712, Durham, NC 27710, દ́tats-Unis.

V. Combes, G.E. Grau :

Département of Pathologie,

K25, Faculté de Médecine,

Université de Sydney,

Sydney NSW 2402, Australie.

ggrau@med.usyd.edu.au

parasitées à la surface de l'endothélium activé. En présence de TNF comme de $\mathrm{LT}$, le nombre de parasites spécifiques à ICAM-1 qui cytoadhèrent à l'endothélium se trouve significativement augmenté, du fait de l'augmentation du nombre de récepteurs disponibles à la surface de la cellule endothéliale. $\varepsilon$ n revanche, si le LMP-420 est ajouté en même temps que le TNF ou la LT, l'adhérence parasitaire due à ICAM-1 se trouve abrogée.

Une étude réalisée au Kenya par Newbold et al. a montré que l'adhérence des hématies parasitées à ICAM-1 était supérieure chez les enfants atteints de PC [5]. Une baisse de cette cytoadhérence par LMP-420 pourrait donc influencer favorablement l'évolution de la maladie chez les jeunes patients.

Enfin, nous avons testé les effets de LMP-420 sur l'augmentation de la vési- 